\title{
Orientación en el programa de tutoría en secundaria
}

\author{
Guidance in high school tutoring program
}

Ana Karen González-Cisneros ${ }^{a}$

\begin{abstract}
:
Guidance is a polysemic term, present in different study plans and programs, mainly at the high school level. However, its implementation is important in basic education, since at the end of it, young people face different options that will frame their Life Project. For this reason, in this research progress report, the product of a master's thesis, a conceptualization of educational and vocational orientation is presented, as well as the identification of its constituent elements at the level of Mexican basic secondary education. For a better understanding, a contextualization is offered on the educational reforms that frame the approaches of educational orientation at that educational level. It is concluded on the importance of identifying the demands of the tutorial activity for the teachers, students and family involved in secondary education.
\end{abstract}

Keywords:

Education, vocational and educative guidance, tutoring

\section{Resumen:}

La orientación es un término polisémico, presente en distintos planes y programas de estudio, principalmente en nivel bachillerato; sin embargo, su implementación tiene importancia en la educación básica, debido a que al concluir la misma los jóvenes se enfrentan a diferentes opciones que van a enmarcar su proyecto de vida. Es por ello que en el presente reporte de avances de investigación, producto de una tesis de maestría, que se presenta una conceptualización de la orientación educativa y vocacional, así como la identificación de elementos constitutivos de ésta en el nivel de la secundaria básica mexicana. Para una mejor comprensión, se ofrece una contextualización sobre las reformas educativas que enmarcan los enfoques de la orientación educativa en dicho nivel educativo. Se concluye sobre la importancia de identificar las demandas de la actividad tutorial para los docentes, alumnos y familia implicados en la educación secundaria.

\section{Palabras Clave:}

Educación, orientación educativa y vocacional, tutoría 


\section{Introducción}

Los alumnos al concluir con su educación básica se encuentran con un panorama amplio dentro del cual deben tomar decisiones informadas que los guíen y orienten hacia un proyecto de vida, de ahí la relevancia de la orientación educativa y vocacional en las aulas desde edades tempranas.

En el presente escrito constituye un reporte parcial de investigación, que se ubica en el proceso de construcción de la tesis del grado de la Maestría en Ciencias de la Educación, por lo que se ofrece una revisión sobre la conceptualización de la orientación educativa como una estrategia de apoyo para los estudiantes del nivel de secundaria básica; se abordan algunos elementos propios de ésta, en los que destacan en los programas de tutoría; para finalizar, se exploran las reformas educativas que han servido como pauta para implementar la orientación y la tutoría en dicho nivel educativo, sobre las que se hace una serie de reflexiones respecto a los ámbitos que en ellas trabaja y cómo se han ido transformando desde que se incluyeron en la educación básica.

\section{¿A qué se refiere la orientación?}

Tomando como base la revisión de la literatura respecto al campo que nos ocupa, se puede llegar a la conclu sión de que el término de orientación es polisémico. Esta afirmación se puede sostener considerando que hay distintas variables y aditamentos para caracterizar el término; para diferenciarlo es necesario considerar el ámbito donde se pretenda aplicar.

Entre las diferentes concepciones se identifican algunos elementos clave para entenderla, entre ellos, por ejemplo, identificamos que Molina (2002) y Buele (2012) coinciden en que se trata de un proceso que tiene repercusiones en el individuo y en su entorno, ya que es integral, interdisciplinario y transdisciplinario. Estas concepciones se caracterizan también, por hacer mención sobre la presencia de una persona con mayor experiencia, que es la que asesora a otra para la solución de problemas (Afonso, 2013).

La orientación, con el añadido de "educativa", habla sobre la aplicación de la misma en un ámbito académico dentro de un plan educativo y considerando a todos los agentes que intervienen en ella (Molina, 2002; Martín ez, 2013 y SEP, 2010-2012). Tanto Denyz Molina como Ascensión Martínez puntualizan algunas características de la orientación educativa, entre ellas se destaca el desarrollo personal, social y profesional, como finalidad, y la intervención de los distintos agentes tanto educativos como sociales que están implicados para su logro.

Se ha relacionado a la orientación educativa con aquella que tiene el añadido de psicopedagógica y que además, está íntimamente vinculada con la dimensión emocional de los sujetos. Bisquerra (2006) se refiere a este tipo de orientación mencionando que se trata de un proceso de ayuda y acompañamiento global de la persona, para favorecerla en el desarrollo personal y a lo largo de toda su vida, siendo ésta una concepción bastante amplia que abarca todos los aspectos de la persona.

Por otro lado, y de acuerdo con la Organización Internacional del Trabajo (OIT) (2016), la orientación vocacional es un servicio que se encuentra ligado a la educación y cuya finalidad es orientar a un individuo para elegir una carrera o profesión, a partir de la evaluación de la compatibilidad entre sus intereses y aptitudes para desarrollarse en lo laboral, destacando la importancia de elaborar un autoconcepto durante el proceso.

En sintonía con los planteamientos anteriores, Molina (2002) y Martínez (2013) mencionan que es aquella que ayuda a que la persona orientada pueda tomar decisiones que le sirvan para que exista un progreso en su vida tanto estudiantil como laboral, a partir del desarrollo de una imagen integrada de sí misma y el rol que puede llegar a ocupar en el mundo del trabajo.

Por ello, la orientación en una acepción general que hace referencia a un proceso en el que no sólo interviene el individuo orientado sino también una persona competente que haga la acción de orientarlo para su desarrollo personal, social y profesional. La orientación educativa se puede dar en distintos lugares, pero es de vital importancia que se dé en un entorno escolar.

En específico, la orientación educativa y vocacional es un proceso que se desarrolla dentro de la educación formal, cuyo objetivo es ayudar al alumno a conocerse a sí mismo, a identificar sus habilidades, destrezas y potencialidades, para que a partir de esto se pueda promover el autoconocimiento y la toma de decisiones encaminadas a hacia la elección de una carrera o una profesión enmarcada en un proyecto de vida.

La educación secundaria en México consta de tres añ os y es el nivel con el que se concluye la educación básica. Al culminar estos tres años, los adolescentes de entre 12 y 15 años de edad, deben tomar decisiones que encaminen su vida, ya sea para continuar estudiando y tener una profesión o para prepararse en un oficio para estar inmersos en el mundo laboral.

De ahí que la orientación educativa y vocacional resulte de suma importancia en el acompañamiento de los alumnos. Sin embargo, no todas las escuelas secundarias cuentan con este servicio educativo, 
aunque es posible encontrar en su lugar y de manera formal, un espacio de Tutoría.

El denominado espacio tutoría comparte elementos que caracterizan a la orientación educativa y vocacional, por lo que también es imperante reconocerlos para darles la importancia que merecen.

\section{La tutoría vista desde las reformas educativas}

La tutoría tiene relativamente poco tiempo de que volvió a aparecer dentro de la educación formal en nivel secundaria. Para contextualizar su presencia en los últimos años, es preciso analizar desde la política educativa las reformas del país en las que está presente.

En 2006, con la entrada al gobierno de Felipe Calderón (panista), se continúa con el proceso de reformas educativas iniciado en el sexenio 2000-2006 con Vicente Fox (también del mismo partido). El primer nivel educativo reformado fue el Preescolar, en 2004, siguiendo con la educación Secundaria en 2006, para finalmente llegar a la educación Primaria en 2009. Todo este recorrido culminó con la Reforma Integral de Educación Básica (RIEB) en 2011.

En el periodo sexenal del presidente Enrique Peña Nieto (priista) (2012-2018) ocurren grandes cambios en educación, que trasciende hasta el año 2017, con el Modelo Educativo centrado en los aprendizajes clave para la educación integral. Esta reforma contempla a toda la educación básica, en la que se incluye el nivel de secundaria, en cualquiera de sus modalidades.

En este periodo de transición en la educación básica, es de interés para la temática que nos ocupa, considerar el espacio curricular que surge en 2006 bajo el título de Orientación y Tutoría, cual es transformado en la reforma del 2011, en la que queda solamente el nombre de Tutoría. No obstante, para el Modelo Educativo de 2017, este espacio no sólo permanece sino que además de Tutoría se le agrega el nombre de Educación Socioemocional, quedando entonces como: Tutoría y Educación Socioemocional.

En primer lugar, tras la implementación de la Reforma Integral de la Educación Secundaria (RIES) en 2006, hubo un trabajo de análisis y reflexión sobre las implicaciones del espacio de Orientación y Tutoría, lo cual dio como resultado algunas modificaciones a los lineamientos generales para su implementación en la RIEB en 2011. Entre los documentos que emitió la Secretaría de Educación Pública (SEP) en 2011, están los lineamientos para la formación y atención de los adolescentes, en los que considera oportuno modificar el nombre del espacio curricular de Orientación y Tutoría, simplificándolo sólo Tutoría, debido a la necesidad de hacer una diferenciación entre ésta y la orientación, ya que en algunas instituciones está presente la figura de un orientador educativo.

La reforma que tuvo lugar en 2017, añade la educación socioemocional al espacio de tutoría, sin embargo, no existe una justificación puntual sobre su cambio dentro de los planes y programas de estudio.

Es así que con la revisión de las reformas educativas y sus cambios en la perspectiva de la orientación y tutoría en educación, que se llega a la conclusión de que, para los fines del trabajo de investigación, se hará referencia al programa como Tutoría, debido a que actualmente se encuentran vigentes tanto el programa proveniente de la reforma 2011 como el de la de 2017, considerando que la implementación para el cambio es gradual.

El espacio de Tutoría, establecido desde 2006, trabaja cuatro ámbitos de intervención del tutor, los cuales son conocidos en las siguientes reformas, como ámbitos de acción tutorial. En general, estos han permanecido, aunque con algunas modificaciones.

Algo que es importante resaltar, es el grado de profundidad que se desarrolla en cada reforma, siendo el programa 2006 mucho más específico que los que le siguen, esto al realizar un ejercicio de comparación de los programas en las dos reformas subsecuentes. Aun así, en los tres programas se habla de una apertura y flexibilidad, con sugerencias para el docente, pero sin ser estrictas en cuanto a organización. En la tabla 1 se muestra la transformación en cuanto al nombramiento de cada ámbito en las tres reformas, también se identifica el cambio para dirigirse a los estudiantes a lo largo de la implementación de éstas.

Tabla 1. Transformación de la orientación y tutoría en tres Reformas Educativas

\begin{tabular}{|c|c|c|c|}
\hline \multicolumn{4}{|c|}{ Reformas Educativas } \\
\hline \multirow{2}{*}{ Ámbito } & \multicolumn{3}{|c|}{ Reformas } \\
\hline & 2006 & 2011 & 2017 \\
\hline 1 & $\begin{array}{l}\text { La inserción } \\
\text { de los } \\
\text { estudiantes en } \\
\text { la dinámica de } \\
\text { la escuela }\end{array}$ & $\begin{array}{l}\text { Integración } \\
\text { entre los } \\
\text { alumnos y la } \\
\text { dinámica de la } \\
\text { escuela }\end{array}$ & $\begin{array}{l}\text { Integración de los } \\
\text { estudiantes a la } \\
\text { dinámica de la } \\
\text { escuela }\end{array}$ \\
\hline 2 & $\begin{array}{l}\text { El seguimiento } \\
\text { al proceso } \\
\text { académico de } \\
\text { los estudiantes }\end{array}$ & $\begin{array}{l}\text { Seguimiento } \\
\text { del proceso } \\
\text { académico de } \\
\text { los alumnos }\end{array}$ & $\begin{array}{l}\text { Acompañamiento } \\
\text { en el proceso } \\
\text { académico de los } \\
\text { estudiantes }\end{array}$ \\
\hline 3 & $\begin{array}{l}\text { La convivencia } \\
\text { en el aula y en } \\
\text { la escuela }\end{array}$ & $\begin{array}{l}\text { Convivencia } \\
\text { en el aula y en } \\
\text { la escuela. }\end{array}$ & $\begin{array}{l}\text { Convivencia en el } \\
\text { aula y la escuela }\end{array}$ \\
\hline 4 & $\begin{array}{l}\text { Orientación } \\
\text { académica y } \\
\text { para la vida }\end{array}$ & $\begin{array}{l}\text { Orientación } \\
\text { hacia un } \\
\text { proyecto de } \\
\text { vida. }\end{array}$ & $\begin{array}{l}\text { Orientación hacia } \\
\text { un proyecto de } \\
\text { vida }\end{array}$ \\
\hline
\end{tabular}
SEP, 2017: 176

De acuerdo con la tabla 1, en 2006, cada ámbito responde a una justificación y a tareas delimitadas hacia 
la escuela, haciendo referencia al primer ámbito, las tareas van en torno a la adaptación de los estudiantes a la dinámica escolar. No obstante, en 2011 este ámbito hace énfasis en la integración de los alumnos, tomando en cuenta la diversidad de estos y que las acciones que se toman al respecto ayuden a contrarrestar la deserción escolar, es decir, la meta es mucho más específica. En cambio, para 2017 se centra en ayudar a los estudiantes a conocer la dinámica de la escuela y acompañarlos en la transición y adaptación que requiere el tránsito de tener un profesor general para todas las asignaturas en la educación primaria a tener un profesor por cada asignatura en la educación secundaria.

Se puede observar que existen modificaciones en cuanto al nombramiento del ámbito, así como al enfoque del mismo en cada momento y la especificidad en cuanto formas de ayudar o incorporar a los estudiantes para lograr los propósitos de la tutoría. De manera medular, permanece el apoyo a los alumnos para que conozcan la dinámica de la escuela y su organización para lograr una adaptación en el tránsito de niveles escolares.

En el segundo ámbito, es importante resaltar que, en la última reforma, aparece como "Acompañamiento en el proceso académico", en donde ya no sólo se le observa como seguimiento, en el sentido de vigilancia, sino que el cambio en la denominación de seguimiento por acompañamiento, implica compartir con el estudiante sus quehaceres y sentires en la escuela. De igual manera, se puede observar cómo inicialmente se refiere a los estudiantes con este nombre para después pasar a denominarlos como alumnos y, en la última reforma, regresa a llamarlos estudiantes tanto en el primero como en el segundo ámbito; las reformas no explican a qué obedece este cambio de nombramiento, sin embargo, sí hay un modo de intervención claro que se menciona a continuación.

En 2006, a diferencia de las siguientes dos reformas, la tarea principal recae en el tutor, pues éste debe dar un seguimiento académico a los estudiantes, así como buscar estrategias tanto individuales como colectivas para la reflexión y solución de las dificultades con las que se encuentren sus alumnos.

Por el contrario, en la reforma 2011 se apuesta por el fomento de la reflexión desde los alumnos para que ellos mismos puedan identificar sus dificultades en las diversas asignaturas $y$, con esto mejorar el aprovechamiento académico, asumiéndose como responsables de la adquisición de sus aprendizajes y dirigiendo estos a lo largo de su vida.

Por último, en 2017 se propone orientar a los estudiantes para el conocimiento de las áreas de oportunidad. La diferencia en estos dos momentos es que se busca fomentar la motivación y el compromiso con el aprendizaje para favorecer el desempeño académico, lo cual no se menciona en la reforma anterior.

Para el tercer ámbito -la convivencia-, las modificaciones son sutiles en cuanto a nombre, sin embargo, el énfasis que se le da en las tres reformas es distinto; en primer lugar, en 2006 se promueven valores como la solidaridad y respeto, para que con estos se tome en cuenta a todos los que conforman la comunidad escolar. La generación de un ambiente con estas características es imprescindible para mejorar el estudio y el aprendizaje. Para 2011, se reconoce y valora la diversidad dentro del aula para a partir de ello, promover el diálogo y la resolución de conflictos de manera pacífica; en este espacio se ve al trabajo de tipo colaborativo como una oportunidad para la formación y el desarrollo grupal y personal, promoviendo la sana convivencia entre los adolescentes.

En 2017, a diferencia de las dos anteriores, se habla acerca de cómo las relaciones en el aula y la escuela pueden influir en los procesos de aprendizaje haciendo hincapié en cómo, en ocasiones, se minimiza el alcan ce que tiene este ámbito, pues se interviene en él para evitar comportamientos disruptivos y no tanto para explotar el potencial formativo con el que cuenta.

El cuarto y último ámbito, tiene un cambio de nombre para la reforma 2011 pero permanece sin modificaciones para la 2017. Respecto a lo establecido en 2006, se habla de una relación entre dos actores que son el tutor y el orientador, los cuales cuentan con la responsabilidad de hacer sugerencias a los alumnos sobre posibles caminos académicos y profesionales, de modo que tengan las herramientas para forjar un plan de vida. En 2011 se menciona el desarrollo del autoconocimiento en los estudiantes para que coadyuve con su capacidad de elección, orientándolos a trazar un plan de vida con base en la reflexión de sus acciones en distintos ámbitos; como se puede observar, queda de manifiesto nuevamente que la responsabilidad recae en el estudiante, a diferencia de la reforma anterior en donde el actor principal es el tutor.

En cuanto a la reforma de 2017, se habla sobre la intervención del tutor a lo largo del ciclo escolar para generar la reflexión de los alumnos en aras de desarrollar un proyecto de vida, a partir de metas a corto, mediano y largo plazo, para que obtengan un panorama sobre cómo las decisiones que tomen en distintos momentos de su vida influirán en el logro de sus proyectos y propósitos a futuro.

Para concluir, se puede observar cómo en un primer momento el trabajo recae en el docente tutor, haciéndole responsable por el apoyo en la toma de decisiones de los alumnos, al brindarles información tanto de la escuela para su mejor convivencia y 
adaptación, como de las opciones que tienen al concluir el momento educativo (su educación secundaria); en las reformas siguientes se busca generar una autonomía en los alumnos para llevarlos a la reflexión y toma de decisiones informadas, pero partiendo de ser responsables de sus acciones y sus procesos de aprendizaje.

Se rescata también el nivel de especificación en cada una de las tres reformas, pues en un primer momento se ofrecen actividades puntuales, así como maneras de intervención en cada ámbito, lo cual es favorable para los docentes al estar a cargo de un espacio sin precedentes. Estas actividades van siendo menos explícitas en la secuencia de reformas, por lo cual la intervención y el fomento de los ámbitos quedan difusos.

\section{Elementos de la orientación}

Una vez identificados los ámbitos de acción tutorial, hay que precisar los elementos de la orientación educativa y vocacional dentro de los planes y programas de estudio, específicamente los que figuran en el Libro para el maestro, de Tutoría y educación socioemocional (2017) expedido por la Secretaría de Educación Pública. Los elementos son los siguientes cuatro:

1. Autoconocimiento

2. Orientación hacia un proyecto de vida

3. Toma de decisiones

4. Acompañamiento en el proceso académico.

De estos elementos, se ofrece una conceptualización, así como la ubicación que tienen en el programa de Tutoría, los cuales iremos describiendo a continuación.

\section{Autoconocimiento}

Si bien es difícil conceptualizar el autoconocimiento, se ha podido hacer una aproximación al término a partir de la revisión documental, considerando concepciones tanto filosóficas como didácticas, en las que se destaca la importancia de este elemento en la formación académica y por ende, en los procesos de enseñanza y aprendizaje que tienen lugar en las aulas de distintos niveles educativos.

Para esto, Lazos (2008) refiere al autoconocimiento como aquel que tiene una persona de sí misma, sobre su propia interioridad, que incluye el conocimiento de hechos relevantes de un sujeto. Aunque el autor no deja de lado lo exterior, se enfoca principalmente en aspectos mentales y psicológicos, a los que la persona tiene un acceso inmediato.

Para que el autoconocimiento sea factible, son necesarios dos niveles de competencia. En primera instancia el desapego, con el cual una persona puede emitir juicios de la manera más objetiva posible sobre sí misma y por otro lado, el involucramiento, que refiere a lo contrario, tomar todos los elementos disponibles para tener una perspectiva única de sí mismo, algo que una persona externa no podría identificar.

Lo anterior se ve respaldado por Martínez (2007), debido a que identifica al autoconocimiento como un proceso de crecimiento en el cual se lleva a cabo una interacción sujeto-medio. Dentro de esta interacción se va transformando el concepto de sí mismo y a la vez, se relaciona con las valoraciones otorgadas por otras personas. Es decir, se ven involucrados los factores intrínsecos y del medio en el que se desenvuelve la persona.

Por otro lado, se destaca que aunque el autoconocimiento se basa en las creencias que una persona tiene sobre sí misma, esto no quiere decir que deban ser ciertas; el proceso de auto-conocerse trata también de identificar concepciones falsas, en ocasiones se puede lograr esto tomando como base lo que piensa o cree otra persona con autoridad sobre uno mismo, ya que se acepta lo que esta figura dice tomándola como una fuente de evidencia confiable.

Lo anterior implica una influencia en la integridad psicológica de una persona (Lazos, 2008), así que para quien influya de este modo, es indispensable tener la capacitación y habilidad de guiar y/o canalizar a la persona que tiene dificultades durante el proceso, que puedan afectar negativamente su integridad psicológica. Desde la perspectiva de La Herrán (2004), existen peligros en caso de que se genere un mal autoconocimiento o mala concepción del mismo. Entre tales advertencias, destaca una desorientación axiológica y una baja evolución personal, lo cual afecta directamente al plan de vida del sujeto.

Para que el autoconocimiento tenga lugar, deben in cidir diferentes factores en la persona, entre ellos, está la organización, la planeación, así como la autoinstrucción, la motivación y la evaluación. Estos procesos ayudan a que se potencie el autoconocimiento. Junto con el razonamiento, el autoconocimiento va a permitir que el estudiante, en específico, entre en procesos reflexivos que abonen a la resolución de problemas (SáizManzanares y Pérez, 2016).

Como parte de la revisión realizada, para el trabajo de investigación se ha optado por considerar al autoconocimiento como aquellas características que un sujeto identifica en sí mismo como propias de su personalidad, tales como aspectos mentales y psicológicos, la manera de actuar ante ciertas situaciones, así como, el razonamiento y la resolución de conflictos. Se toma en cuenta que para que éste tenga lugar, se necesitan diferentes factores y la ayuda de agentes externos que aporten información sobre la persona, pero que además, la orienten en el proceso de construcción de una imagen de sí mismo. 
Cabe señalar que se hace explícita la relevancia de considerar su inclusión y promoción en los planes y programas de estudio, debido a la importancia que tiene para el estudiante la resolución de problemas, la toma de decisiones y el apoyo a la formulación de un plan de vida.

\section{Ubicación en el programa}

De los cuatro elementos rescatados de la orientación educativa y vocacional, el autoconocimiento es el que tiene mayor presencia y fuerza dentro de los planes y programas de estudio de la SEP, debido a que conforma una de las dimensiones de la educación socioemocional, y por lo tanto se encuentra de manera reiterada en diversos espacios de los documentos revisados. Además de que mantiene una relación constante con los otros elementos y con diferentes componentes de la tutoría.

En primer lugar, el perfil de egreso de educación básica (que culmina con la educación secundaria) establece que los alumnos deben poseer autoconocimiento y regular sus emociones. En este punto se establece que los egresados, deben procurar su bienestar en diferentes momentos de su vida, a corto, mediano y largo plazo, además de entender el concepto de proyecto de vida, para que esto favorezca en la elaboración de sus planes personales.

Como se puede observar, el autoconocimiento viene acompañado de otro concepto central para la orientación, que es el de plan de vida, además de hacer referencia a la toma de decisiones para los planes personales (SEP, 2017: 101). Es importante resaltar que dentro de los ámbitos de acción del espacio curricular de tutoría se establece, como se verá más adelante, el de Orientación hacia un proyecto de vida, que en el plan y programas de estudios de la reforma educativa de 2017 , se encuentra vinculado directamente con la dimensión de autoconocimiento. Es decir, las dos dimensiones propias de la Orientación Educativa y Vocacional están inmersas y trabajan en conjunto en el sistema educativo. Es importante mencionar que se trata de un trabajo continuo, que inicia en el preescolar y culmina en el nivel de secundaria; en donde los ambientes de aprendizaje deben promover la formación integral de los estudiantes y su capacidad de autoconocimiento, asumiendo que cada uno cuenta con características diversas, lo cual debe estar contemplado en el trabajo escolar. De esta manera, se busca que los estudiantes sean favorecidos, fortaleciendo con ello las estructuras de conocimiento con las que cuentan y dando lugar al aprendizaje significativo (SEP, 2017: 124).

El autoconocimiento no sólo se busca en el espacio curricular de Tutoría y Educación Socioemocional, sino que se incluye en otros dos espacios pertenecientes al área de desarrollo personal y social que son, las Artes y la Educación Física (SEP, 2017: 159).

\section{Educación socioemocional}

La educación socioemocional, presente a lo largo de toda la educación básica, contempla cinco dimensiones: el autoconocimiento, la autorregulación, la autonomía, la empatía y la colaboración, las cuales se presentan en la figura 1. El autoconocimiento toma el primer lugar en estas dimensiones y éstas se han de trabajar, no sólo en la hora semanal designada en la carga curricular para nivel secundaria, sino que deben estar presentes en todo el espacio escolar de este nivel (SEP, 2017: 167).

\section{Figura 1. Educación socioemocional y sus dimensiones}

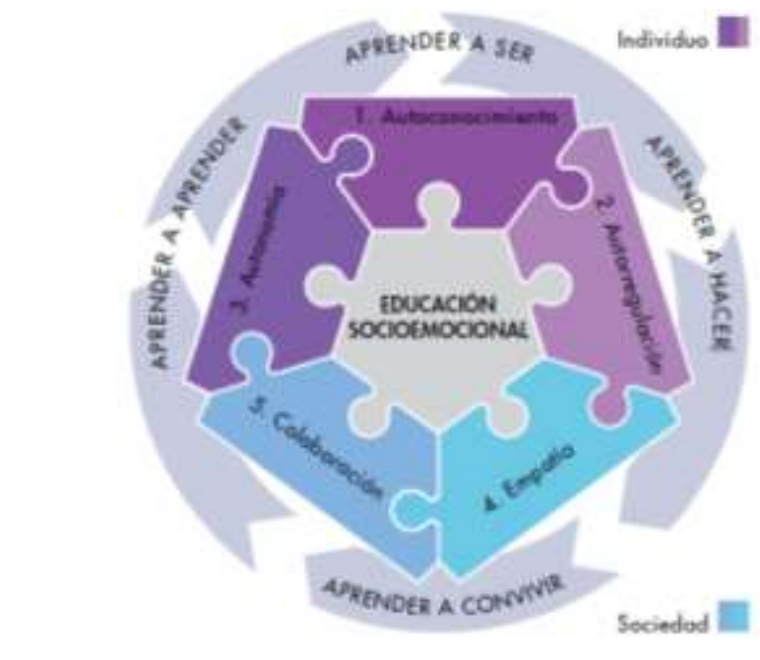

Fuente: Libro para el maestro (SEP, 2017: 181)

El autoconocimiento no sólo se encuentra como eje rector en las dimensiones, sino que también se establece como el primer propósito de la educación socioemocional, y aún más específico, se encuentra también como primer propósito de esta área de conocimiento para la educación secundaria, en la que se busca el fortalecimiento del autoconocimiento y la autorregulación para favorecer la atención, en donde el tutor debe fungir como acompañante para lograrlo (SEP, 2017: 172).

Como dimensión del área de educación socioemocional, es importante destacar que el autoconocimiento tiene niveles de logro, mismos que sirven como alternativa para la evaluación en este espacio, y es donde se puede ver la importancia que tiene en la educación básica. Aunado a los niveles de logro, se encuentran habilidades específicas que se asocian con las dimensiones socioemocionales, la cuales son: atención, consciencia de las propias emociones, autoestima, aprecio y gratitud, así como bienestar. A partir de éstas, se establece el nivel de logro de los alumnos por grado, 
que aparecen registrados en las figuras 2, 3 y 4 de acuerdo con los tres grados de la educación secundaria.

Figura 2. Educación socioemocional en primero de secundaria

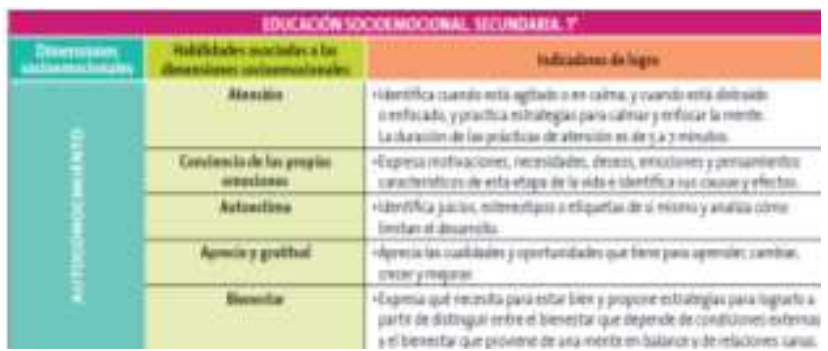

Fuente: Libro para el maestro (SEP, 2017: 214)

Figura 3. Educación socioemocional en segundo de secundaria

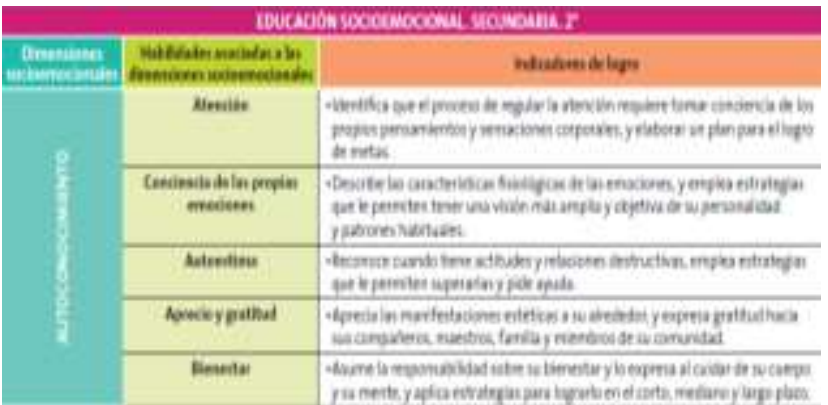

Fuente: Libro para el maestro (SEP, 2017: 215)

Figura 4. Educación socioemocional en tercero de secundaria

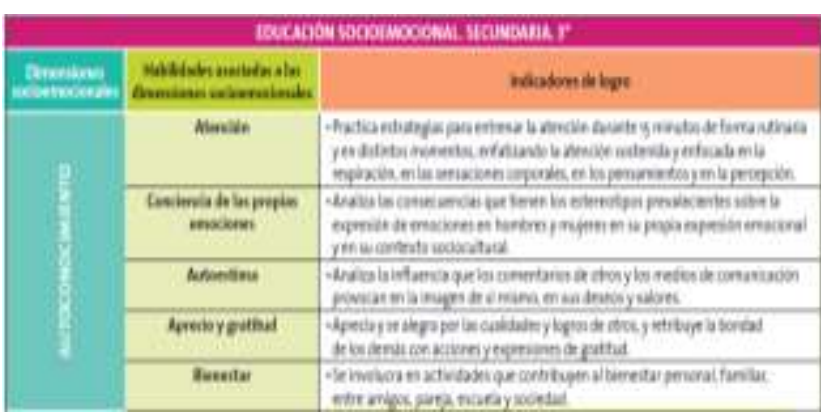

Fuente: Libro para el maestro (SEP, 2017: 216)

El autoconocimiento tiene cabida en la educación debido a que forma parte del razonamiento y junto con éste, es esencial para la resolución de problemas que se pueden presentar tanto en el contexto escolar como en la vida cotidiana. Para esto es necesario además, que el alumno cuente con autorregulación. En el proceso de resolución de problemas se encuentran distintas fases, en primer lugar, existe la de monitoreo, en la que se relaciona la tarea o la situación con los conocimientos previos. Posterior a ella, se establecen metas para la correcta ejecución y al final, se analiza la respuesta dada, promoviendo una reflexión de la acción, lo cual es importante, pues a partir de esto y de ser necesario, la respuesta se modifica. Los procesos inmersos en la autorregulación, al momento de resolver problemas, potencian el autoconocimiento del alumno (SáizManzanares y Pérez, 2016).

El autoconocimiento es el elemento con mayor presencia en el programa para tutorías de la SEP, debido a que se establece como dimensión de trabajo que implica el involucramiento en los diversos ámbitos de la acción tutorial, como se mencionó en líneas anteriores y, por ende, en habilidades por desarrollar en los alumnos.

\section{Orientación hacia un proyecto de vida}

Sobre los proyectos de vida, D'Angelo (2006) los identifica desde una perspectiva psicológica y social, como una estructura a partir de la cual una persona puede expresar una dirección hacia el futuro, además de la atención a ciertas áreas que requieren la toma de decisiones vitales. El proyecto de vida se relaciona y depende de la situación social actual del individuo y de su perspectiva a futuro.

El proyecto de vida se presenta como una tarea fundamental en los espacios concernientes tanto a la orientación como a la tutoría, tal como se puede identificar en los programas de estudio de la SEP y como lo menciona Krichesky (1999), la asistencia para la elaboración de un proyecto de vida es una de las principales funciones de programas de esta naturaleza; idea que es reforzada por De León y Rodríguez (2008), quienes señalan la importancia de que otras personas ayuden al estudiante a forjar un proyecto de vida como parte de los programas de orientación vocacional.

\section{Ubicación en el programa}

La orientación hacia un proyecto de vida es uno de los cuatro ámbitos de la acción tutorial, por tanto, establece un trabajo continuo durante todo el ciclo escolar, tal como se encuentra plasmado en el Libro para el maestro de secundaria (SEP, 2017: 178). Sin embargo, éste no pone de manifiesto un propósito, pero menciona el papel del tutor como persona que interviene mediante la reflexión con los alumnos, en las metas que éstos se plantean a corto, mediano y largo plazo, así como sobre las implicaciones que tienen sus decisiones y acciones para momentos futuros, las cuales impactarán en el logro de sus proyectos y sus propósitos.

El proyecto de vida se relaciona con la dimensión de autoconocimiento, que es de gran importancia en la orientación, en la de autorregulación y en la autonomía. Esto debido a que se propone un trabajo para que los alumnos reconozcan sus intereses, capacidades y potencialidades, y con ello, puedan tomar decisiones tanto en momentos de su vida inmediata como a futuro. La implementación de este ejercicio se destaca desde el 
primer grado de la educación secundaria, pues, la construcción de un proyecto de vida es algo complejo que se va forjando a partir de distintos procesos de conocimiento, análisis, reflexión, toma de decisiones y, finalmente, con la ejecución de ciertas acciones que favorezcan al mismo.

De la misma manera, el Libro para el maestro establece que, una vez que los alumnos son orientados por el docente en cuanto a sus fortalezas, habilidades y también dificultades, que forman parte de un balance personal, se establecen posibles escenarios en los cuales puedan desarrollarse, para después conducirlos a la búsqueda de información más precisa sobre los perfiles formativos a los cuales se inclina, siempre procurando un bienestar (SEP;2017: 179).

\section{Toma de decisiones}

La toma de decisiones es otro de los elementos de la orientación educativa y vocacional, que está presente en el programa. Sin embargo, de los cuatro elementos identificados éste es el que tiene menor información puntual, ya que a diferencia de los otros, no conforma un ámbito o dimensión, que son los segmentos de mayor organización dentro del programa. La toma de decisiones se encuentra junto con el compromiso y es tomada como una habilidad dentro de la dimensión de la autonomía. Además de esto, se encuentra en los ámbitos y dimensiones de la educación socioemocional y del programa de tutoría.

Sobre la toma de decisiones como habilidad, se establece que hay una vinculación con la autonomía moral y debe ejecutarse en función de lo que beneficia o perjudica tanto al alumno como a los que se encuentran a su alrededor. También se resalta el papel del tutor para que la toma de decisiones se lleve a cabo a partir de la reflexión.

Dentro del rubro de la toma de decisiones, es muy importante resaltar los cambios estructurales que se dan a nivel cerebral durante la adolescencia, ya que con ello es que se justifica la implementación de la tutoría en secundaria. Durante el periodo de la adolescencia existen cambios en la corteza prefrontal, que intervienen en la organización de los procesos mentales, como el pensamiento y la propia toma de decisiones, debido a que permite la evaluación de una situación, para posteriormente, racionalizarla, recordar otros acontecimientos y reflexionar sobre lo que sucede (SEP, 2017: 170).

\section{Acompañamiento en el proceso académico}

Los espacios de orientación o tutoría, dentro de un ambiente escolar, están inherentemente conectados con el acompañamiento académico, debido al constante cambio de la educación de carácter formal y para satisfacer las necesidades de una sociedad en transformación. Por ello es necesario hacer una mención puntual de lo que implica el acompañamiento académico en los espacios escolares y a qué se refieren.

El proceso de acompañamiento tiene distintas ventajas, pero en esencia, tiene un carácter preventivo, además de actuar como facilitador del desarrollo de competencias en los estudiantes (Araiza, 2004). El acompañamiento académico puede ser una estrategia para abatir la deserción escolar y canalizar a los estudiantes que presentan dificultades en diferentes ámbitos de su vida, y que puedan afectar directamente en su desempeño académico, para con ello favorecer tal desempeño.

La importancia del acompañamiento se encuentra en distintos niveles educativos, se puede observar, por ejemplo, que la formación profesional se hace de tal manera que los egresados carecen de herramientas y habilidades que les permitan responder a las necesidades de la sociedad o bien, no prever que el mercado laboral es escaso y de cualquier modo no pueden desempeñarse en cierto ámbito. En este sentido, el acompañamiento en el proceso académico juega un papel importante, pues es un factor que ayu da a vislumbrar estas situaciones y capacitar a los estudiantes para que al concluir su educación formal, estén listos para acceder al mundo laboral. De este modo, los profesores se ven en la necesidad de brindar un acompañamiento a los estudiantes para garantizar una educación de calidad que los prepare para la vida en sociedad (Araiza, 2004).

Por su parte, Tilano, Henao y Restrepo (2008) identifican la influencia que tiene la familia en el desempeño académico y por ende, la importancia de que el vínculo que se genere de ésta con la escuela sea favorable para el alumno. Dada la relevancia de la familia, es imprescindible el conocimiento sobre la dinámica de los alumnos en ella, para poder realizar una intervención o canalización y, de ser necesario, fungir un papel de orientador en sus procesos académicos para la mejora de los resultados, lo cual es propio del acompañamiento académico.

Se han documentado proyectos que, entre otras cosas, se encargan de brindar un acompañamiento académico a alumnos en diversas situaciones, como es el de RuízRomán (2018), que entre las actividades realizadas, encuentra a la tutoría individualizada tanto con los jóvenes como con sus familias, como herramienta que tiene como finalidad tratar cuestiones con respecto a la trayectoria académica de los estudiantes. También se ejercen algunas actividades de apoyo al estudio, que son coordinadas por otras instancias; se da seguimiento al desempeño del alumno, recabando información 
concerniente a sus calificaciones, reuniones, tutorías e incidencias que puedan tener en la escuela.

\section{Ubicación en el programa}

A diferencia del autoconocimiento, que se encuentra como ámbito de acción y se le menciona de manera puntual dentro del programa, además de que se le relaciona tanto de manera implícita como puntualmente con los otros elementos de la orientación, como son: la toma de decisiones, el autoconocimiento y el apoyo para elaborar un plan de vida, el acompañamiento en el proceso académico es uno de los cuatro ámbitos de la acción tutorial que se trabaja en el espacio de Tutoría en secundaria a lo largo de todo el ciclo escolar.

La presencia del acompañamiento escolar durante todo el ciclo escolar debe estar en diversas actividades. Al respecto, el Libro para el maestro (2017) marca como propósito de este ámbito de acción, que los estudiantes sean orientados para reconocer y analizar su desempeño en cada asignatura, con la finalidad de identificar tanto fortalezas como dificultades $y$, a partir de esto, se puedan desarrollar algunas estrategias que les permitan la adquisición de los aprendizajes esperados.

Se relaciona estrechamente con el autoconocimiento como dimensión socioemocional, y busca fomentar la autonomía de los estudiantes a partir de identificar sus necesidades y tomar decisiones en aras de mejorar su desempeño académico. Lo que hace que este ámbito de acción esté impregnado de características propias de la orientación y se pueda observar cómo se relaciona constantemente la acción tutorial o de orientación con el acompañamiento académico.

\section{Conclusiones}

Existen elementos propios de la orientación educativa y vocacional dentro de los planes y programas de tutoría en educación básica; sin embargo, se da más énfasis al desarrollo de ésta en los niveles educativos posteriores, lo cual es entendible debido a la cercanía que tienen los alumnos para la elección de carrera. No obstante, esta elección se puede fundamentar y apoyar en niveles más tempranos. He ahí la importancia de aprovechar los espacios destinados a esta actividad en la educación básica, para promover la toma de decisiones informadas que posteriormente se emplearán en la vida académica de los estudiantes. Por tanto, estos espacios tendrían que contar con la organización suficiente y la capacitación de los docentes al frente de los mismos, que les permita cumplir con su cometido y, en este sentido, acompañar académicamente a los estudiantes, así como orientarlos en la toma de decisiones que enmarquen su proyecto de vida.
Los ámbitos de acción tutorial, desde su inclusión en el 2006, han permanecido en el espacio curricular de tutoría, aunque con enfoques distintos, pero algo que ha de destacarse es la falta de especificidad, en cuanto a las actividades a desarrollarse 0 al campo temático a atender. Dado que en el espacio curricular es imperan te una serie de necesidades y demandas dirigidas a los docentes, que puede que tengan o no conocimientos al respecto, pero que están dirigidas a cómo tutorar u orientar a los alumnos en la toma de decisiones que enmarquen su proyecto de vida, así como en el proceso de autoconocimiento y todo aquello que englobe el acompañamiento en el proceso académico.

Es por lo antes mencionado, que esta revisión documental y la reflexión que queda abierta a partir de la misma, permite iniciar un proceso de indagación respecto a quiénes son los docentes que están desarrollando el ejercicio tutorial y de orientación en este momento educativo, para con ello conocer su quehacer en el aula, en las actividades de formación extracurricular y la importancia que estos docentes dan a la labor tutorial, para así poder fortalecer o mantener la búsqueda para la transformación o replanteamiento de las reformas educativas abordadas en este texto.

\section{Referencias}

Afonso Ribeiro, Marcelo. (2013). Reflexiones epistemológicas para la orientación profesional en América Latina: una propuesta desde el Construccionismo Social. Revista Mexicana de Orientación Educativa, Vol. 10, Núm. 24, pp. 2-10. http://pepsic.bvsalud.org/scielo.php?script=sci_arttext\&pid=S 166 $5-75272013000100002 \& \operatorname{lng}=$ pt\&tlng=es. [Recuperado 06 de abril de 2018]

Ariza, Gladys Ibeth y Ocampo, Héctor Balmes (2005). El acompañamiento tutorial como estrategia de la formación personal y profesional: un estudio basado en la experiencia en una institución de educación superior. Universitas Psychologica, vol. 4, núm. 1, enero-junio, 2005, pp. 31-41 http://www.scielo.org.co/scielo.php?script=sci_arttext \&pid=S165 7-92672005000100005

Bisquerra Alzina, Rafael (2006). "Orientación psicopedagógica y educación emocional”. ESE. Estudios sobre educación, $\mathrm{N}^{\circ} 11$, pp.9-25. https://dadun.unav.edu/bitstream/10171/9208/1/Ea.pdf

Buele Maldonado, Mariana Angelita (2015). Análisis de las necesidades y servicios de orientación universitaria de los estudiantes de la modalidad abierta y a distancia. Tesis Doctoral. Facultad de Psicología, Universidad de Santiago de Compostela. www.minerva.usc.es > xmlui > bitstream > rep_1047

Cañete Villafranca, Roberto, Guilhem, Dirce, y Brito Pérez, Katia. (2013). Paternalismo médico. Revista Médica Electrónica, Vol. 35, Núm. 2, pp. 144-152. Recuperado en 06 de agosto de 2019, de http://scielo.sld.cu/scielo.php?script=sci_arttext\&pid=S1684$18242013000200006 \& \ln \mathrm{g}=\mathrm{es} \& \ln \mathrm{g}=\mathrm{es}$

D'Angelo Hernández, Ovidio (2006). "Proyecto de vida y desarrollo integral”. Revista Internacional Crecemos, Año 6, Núm. 1 y 2, pp. 
http://biblioteca.clacso.edu.ar/ar/libros/cuba/cips/caudales05/Caud a les/ARTICULOS/ArticulosPDF/07D050.pdf

De la Herrán, Agustín (2004). El autoconocimiento como eje de la formación. Revista Complutense de Educación, Vol. 15 Núm. 1 pp. 11-50. https://revistas.ucm.es/index.php/RCED/article/view/RCED04041 20011A

Grady Patricia A, Lucio-Gough Lisa (2015). El automanejo de las enfermedades crónicas: un método integral de atención. Panam. J. Public. Health. Vol. 35, Núm. 9, pp. 187-194. https://www.scielosp.org/pdf/rpsp/2015.v37n3/187-194

Grañeras Pastrana Montserrat y Parras Laguna, Antonia (Coord.) (2008). Orientación educativa: fundamentos teóricos, modelos institucionales y nuevas perspectivas. España: Centro de Investigación y Documentación Educativa. https://diversidad.murciaeduca.es/orientamur/gestion/documentos/ inv2008oeftminppc.pdf

Krichesky, Marcelo (1999). Proyectos de orientación y tutoría. Paidós. Obtenido

de http://www.terras.edu.ar/biblioteca/16/16TUT_Krichesky_Unidad 3.pdf

Lazos, Efraín (2008). Autoconocimiento: una idea tensa. Revista Diánoia, Vol. LIII, Núm, 61 (noviembre 2008): pp. 169-188. http://www.scielo.org.mx/pdf/dianoia/v53n61/v53n61a6.pdf

León Mendoza, Teresa De y Rodríguez Martínez, Rafael(2008). El efecto de la orientación vocacional en la elección de carrera. Revista Mexicana de Orientación Educativa, Vol. 5 Núm. 13,pp. 10-16. http://pepsic.bvsalud.org/pdf/remo/v5n13/v5n13a04.pdf

Martínez Cano, Amparo (2007). El incremento del autoconocimiento y la toma de decisiones en alumnos de educación secundaria obligatoria, de Cuenca. Revista Española de Orientación y Psicopedagogía, Vol. 18, Núm. 1, pp. 17-30. http://revistas.uned.es/index.php/reop/article/view/1 1294

Martínez Martínez, Asunción (2013). La orientación como actividad educativa y vocacional en los itinerarios curriculares del alumnado de bachiller y formación profesional y su inclusión en el mercado laboral. Tesis doctoral, Universidad de Granada, Facultad de Ciencias de la Educación, Granada. file:///C:/Users/USUARIO/Downloads/22560063.pdf Recuperado el 17 de 02 de 2018

Molina Contreras, Denyz Luz (2002). Concepto de orientación educativa: diversidad y aproximación. Revista Iberoamericana de educación. https://rieoei.org/historico/deloslectores/736Molina 108.PDF

Organización Internacional del Trabajo (OIT, 2016). Manual de servicios de orientación vocacional.

Ruíz-Román, Cristóbal, Molina, Lorena y Alcaide, Rocio (2018). Trabajo en red y acompañamiento socioeducativo con estudiantes de secundaria en desventaja social. Revista de currículum y formación del profesorado, Vol. 22, Núm. 3, pp. 453-474. https://recyt.fecyt.es/index.php/profesorado/article/view/69202

Sáiz-Manzanares, María Consuelo y Pérez Pérez, Magdalena Isabel (2016). Autorregulación y mejora del autoconocimiento en resolución de problemas. Psicología desde el Caribe, enero-abril de 2016. Vol. 33, Núm. 1, pp. 14-30. https://www.redalyc.org/pdf/213/21345152002.pdf

Secretaría de Educación Pública (SEP, 2006). La orientación y la tutoría en la escuela secundaria. Lineamientos para la formación y la atención de los adolescentes. México.

Secretaría de Educación Pública (SEP, 2012). Plan de estudios 2011. Secretaría de Educación Pública.
Secretaría de Educación Pública (SEP, 2017). Aprendizajes Clave. Tutoría y Educación Socioemocional. Educación Secundaria. Ciudad de México.

Secretaría de Educación Pública. (2010-2012). Programa de orientación educativa. México, D. F.

Tilano, Luz Magnolia, Henao, Gloria Cecilia y Restrepo, Julián A. (2009). Prácticas educativas familiares y desempeño académico en adolescentes escolarizados en el grado noveno de instituciones educativas oficiales del municipio de Envigado. El Ágora, Vol. 9,

$\begin{array}{lll}\text { educativas oficiales del municipio de Envigado. El Agora, Vol. 9, } \\ \text { Núm. } & 1, & \text { pp. }\end{array}$ https://www.redalyc.org/pdf/4077/407748994002.pdf 